\title{
Next-generation protein analysis in the pathology department
}

\author{
Melek Ahmed (1) ,' Glenn Broeckx (1) , ${ }^{2}$ Geert Baggerman, ${ }^{3,4}$ Karin Schildermans, ${ }^{4,5}$ \\ Patrick Pauwels, ${ }^{2,6}$ Amaryllis H Van Craenenbroeck, ${ }^{7,8}$ Amelie Dendooven ${ }^{2,6,8}$
}

\begin{abstract}
${ }^{1}$ Faculty of Medicine and Health Sciences, Department of Medicine, University of Antwerp, Antwerp, Belgium

${ }^{2}$ Department of Pathology, University Hospital of Antwerp Antwerp, Belgium

${ }^{3}$ Department of Biology,

University of Antwerp, Antwerp, Belgium

${ }^{4}$ Unit Health, Flemish Institute for Technological Research, Mol, Belgium

${ }^{5}$ Departments of Molecular Imaging, Pathology,

Radiotherapy and Oncology, University of Antwerp, Antwerp, Belgium

${ }^{6}$ Center for Oncological

Research Antwerp (CORE), University of Antwerp, Faculty of Medicine and Health Sciences, Antwerp, Belgium

${ }^{7}$ Department of Nephrology and Renal Transplantation, University Hospitals Leuven, Leuven, Belgium

${ }^{8}$ Laboratory of Experimental Medicine and Pediatrics, University of Antwerp, Antwerp, Belgium
\end{abstract}

\section{Correspondence to}

Professor Amelie Dendooven, Department of Pathology, Universitair Ziekenhuis Antwerpen, Edegem 2650, Belgium; amelie.dendooven@ uantwerpen.be

Received 27 March 2019 Revised 12 June 2019

Accepted 20 June 2019 Published Online First 15 July 2019

\section{Check for updates}

(C) Author(s) (or their employer(s)) 2020. No commercial re-use. See rights and permissions. Published by BMJ.

To cite: Ahmed M, Broeckx G, Baggerman G, et al. J Clin Pathol

2020;73:1-6.

\section{ABSTRACT}

Traditionally, immunohistochemistry (IHC) is used by pathologists to localise specific proteins or peptides in tissue slides. In the era of personalised medicine, however, molecular tissue analysis becomes indispensable for correct diagnosis, prognosis and therapeutic decision, not only on the DNA or mRNA level but also on the protein level. Combining molecular information with imaging presents many advantages. Therefore, matrix-assisted laser desorption/ionisation imaging mass spectrometry (MALDI IMS) is a promising technique to be added to the armamentarium of the pathologist. Here, we focus on the workflow, advantages and drawbacks of both MALDI IMS and IHC. We also briefly discuss a few other protein imaging modalities and give examples of applications.

\section{INTRODUCTION}

The discipline of histopathology has a long history. ${ }^{1}$ Technical improvements have led us from macroscopy over microscopy into molecular analysis. The use of microscopy images for clinical diagnosis lies at the core of the pathologist's profession. Therefore, the pathologist is likely the best-placed medical professional for integrating molecular findings with information from detailed microscopic images. ${ }^{2}$

Over the last years, pathologists have become familiar with molecular techniques for DNA and mRNA analysis from tissue. However, measurement of protein alterations potentially better predicts functional consequences (and thus diagnosis and prognosis) than DNA alterations, because proteins themselves execute biological processes. ${ }^{3}$ Recently, many techniques have been developed for more detailed molecular protein analysis. Especially, matrix-assisted laser desorption/ionisation imaging mass spectrometry (MALDI IMS) emerges as a powerful tool. Virtually no diagnostic pathology laboratories have yet embraced MALDI IMS for dayto-day practice, though this is likely to change in the near future.

This review aims to introduce clinical pathologists to MALDI IMS. In order to outline the advantages of the technique, a comparison with immunohistochemistry (IHC) is made. The workflow of both techniques is summarised, advantages and disadvantages are compared and applications are discussed.

\section{IMMUNOHISTOCHEMISTRY Principle and workflow}

The essence of IHC is to make specific proteins or peptides visible under the microscope by means of antigen-antibody recognition by a specific immunoglobulin or primary antibody. IHC is mostly performed on slides cut from formalin-fixed, paraffin-embedded (FFPE) tissue, on slides generally around $2-5 \mu \mathrm{m}$ thick that are first deparaffinised, washed with xylene and rehydrated through graded ethanol series (100\%, 96\% and 70\%) before being subjected to antigen retrieval and application of antibodies. Heat-induced or enzyme-based antigen retrieval is necessary on FFPE tissue to make the epitopes ready for recognition by the primary antibody. ${ }^{4}$ The primary antibody is either monoclonal (generated via hybridoma technology) ${ }^{5}$ or polyclonal. Monoclonal antibodies have better specificity, while polyclonal antibodies tend to be more sensitive but less specific and give more background staining. The antigen-immunoglobulin interaction is visualised by addition of a conjugate at the primary antibody (direct IHC) or the secondary antibody (indirect IHC). In IHC, this conjugate is an enzyme, usually horseradish peroxidase or alkaline phosphatase. The addition of a chromogenic substrate results in a coloured precipitate. Different secondary antibody detection techniques have been developed over the years, such as the avidinbiotin-peroxidase complex, the labelled streptavidin-biotin complex and polymer-based detection systems. ${ }^{4}$ Polymer-based detection systems allow for good visibility and are now widely used in routine diagnostics. Automatisation techniques enable high throughput for diagnostic use, ${ }^{6}$ using robotisation for pipetting reagents on slides. The workflow of an IHC experiment, including tissue preparation, is outlined in figure 1.

IHC can be performed on cytology specimens, provided adequate tissue preparation is used, ${ }^{7}$ and on frozen sections. ${ }^{4}$

In immunofluorescence (IF), ${ }^{9}$ mostly performed on frozen sections, the conjugate consists of a fluorophore instead of an enzyme.

Some applications and future perspectives of IHC IHC on FFPE is widely used in the pathology laboratory. At the Antwerp University Hospital, on average, two immunostains are performed per case, with a wide variability from 0 to more than 20 stains per diagnostic case.

IHC helps to subtype tumours, or visualises specific structures in the tissue, enabling the pathologist to establish a specific diagnosis. IF studies on skin or kidney tissue are typically used to visualise immunoglobulin or complement deposits. ${ }^{9}$

However, in the era of precision medicine, pathologists are increasingly involved in the evaluation of predictive factors for treatment, especially in 


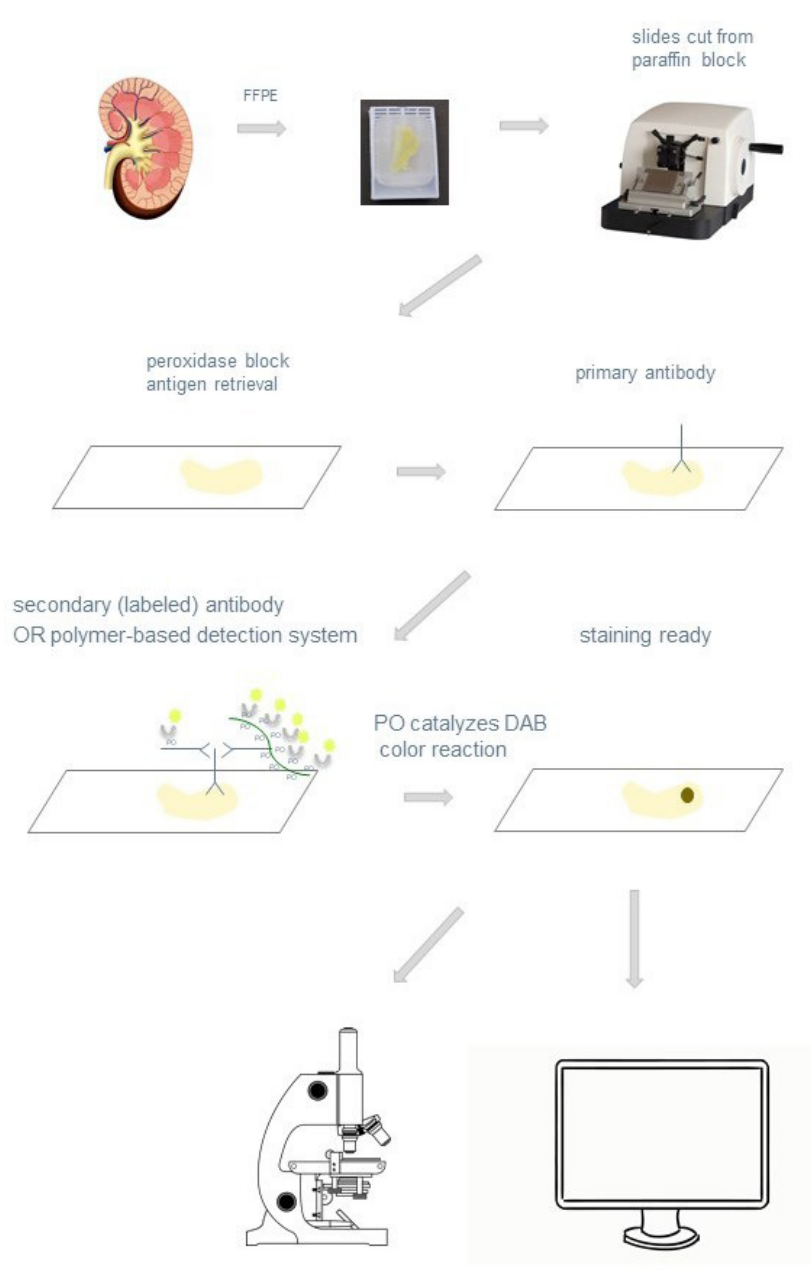

Figure 1 Workflow of immunohistochemistry on FFPE sample: the tissue is FFPE. Tissue sections are cut on a microtome and put on a slide, which is then deparaffinised and rehydrated. After peroxidase block and antigen retrieval (typically performed by boiling or microwaving the tissue in a buffer), the primary antibody is added to the slide. Then, the secondary antibody or polymer-based secondary antibody system is added. A PO moiety is present on this secondary antibody or moiety. This permits the DAB horse radish peroxidase substrate to be oxidised by the $\mathrm{PO}$ and changed into a brown colour on the tissue slide. The brown colour thus indicates the place of binding of the primary antibody with the targeted (protein) antigen. The slide is then interpreted by the human eye under the microscope or analysed through the computer after digital slide scanning (picture of FFPE block: Leica, picture of microtome: chinamedevice.com). DAB, 3,3-diaminobenzidine; FFPE, formalin-fixed, paraffin-embedded; PO, peroxidase.

the oncology field. Not only rendering a specific diagnosis is thus requested but also also the determination of certain prognostic and predictive factors. Examples include the determination of oestrogen and progesterone receptor status in breast carcinoma, ${ }^{10}$ the use of ALK staining for screening for ALK translocation in lung carcinoma ${ }^{11}$ and the determination of $B R A F$ V600E mutation status in melanoma. ${ }^{12}$ Whereas conventional diagnostic decision-making usually includes more contextual clues, these assays guide treatment in a more binomial (yes/no) manner. This obviates the need for stringent quality control measures and validated scoring schemes for predictive immunohistochemical tests. An example of a scoring system is the Allred score for quantification of hormonal receptors in breast cancer. ${ }^{13} 14$
In the very nearby future, variability in human interpretation might benefit considerably from digital image quantification performed on scanned slides. ${ }^{15}$ In 2017, the FDA approved marketing of first whole slide imaging system for digital pathology.

In the field of IF, exploiting physical characteristics have led to the advent of a series of techniques that have improved spatial resolution by limiting diffraction, such as confocal scanning laser microscopy or super-resolution microscopy techniques, such as structured illumination microscopy, stimulated emission depletion microscopy, near-field scanning optical microscopy, saturated structured illumination microscopy, photoactivation localization microscopy and light-sheet microscopy, just to mention a few. ${ }^{16} 17$ These techniques are extremely popular for scientific research and are highly suited for digital quantification, but are currently not often used for day-to-day practice.

\section{MATRIX-ASSISTED LASER DESORPTION/IONISATION IMAGING MASS SPECTROMETRY Principle and workflow}

Mass spectrometry (MS) encompasses a whole set of techniques to analyse different molecules, including, but not limited to, proteins and peptides, based on the molecular mass. MALDI IMS is one of these MS techniques, that additionally boasts the advantage of generating images. ${ }^{18}$ The workflow of a MALDI IMS experiment is outlined in figure 2.

Preparation of a sample requires a tissue section around $5-10 \mu \mathrm{m}$ thick. Preparation steps differ, depending on the tissue used, FFPE or frozen sections. FFPE tissue sections have to undergo deparaffinization and washing with xylene in order to rewax the tissue. Hereafter, rehydration through graded ethanol series $(100 \%, 96 \%$ and $70 \%)$ is needed. Antigen retrieval can be either enzymatic, whereby proteins are cleaved into peptides, or heat mediated, whereby proteins undergo denaturation. These step are similar to the intial steps needed for preparation of FFPE tissue for IHC. Then, trypsin solution is applied with a spraying system. Tissue is digested during 1.5 hours at $37^{\circ} \mathrm{C}$ under 95\% humidity. Enzyme application is a crucial preparation step for FFPE as different approaches can be used (different buffers, different concentrations of enzyme required, different digestion times and temperatures) in order to minimise the time and effort needed and maximise the spatial information. ${ }^{19-22}$

For fresh-frozen (FF) tissues, the preparation step consists of ethanol washing for direct profiling of protein with a mass below $\pm 25 \mathrm{kDa}$. An additional preparation step for high-molecularweight proteins $(> \pm 25 \mathrm{kDa})$ is trypsin application, since these proteins are not easily detected in matrix-assisted laser desorption/ionisation (MALDI) analysis of FF tissues due to their poor ionisation efficiency in this mass range. ${ }^{19} 23$

The tissue is then placed on a target. In the next step, the tissue section has to be coated with a matrix and air-dried. The method of application of the matrix also needs to be optimised, finding the balance between sufficient extraction of the analytes and avoiding lateral diffusion, which is detrimental for the resolution of the obtained images. Different matrices can be used for different types of analytes. The background signal can be quite high, limiting sensitivity of detection for some small molecules.

Then the sample is introduced into the mass spectrometer. During the ionisation process, a laser beam irradiates the matrix. Laser energy is absorbed, causing the transition of the solid matrix and the embedded analytes into a gaseous phase in which the molecules acquire a charge. MALDI is typically coupled to a time-of-flight (TOF) mass analyser. The ionised molecules are 

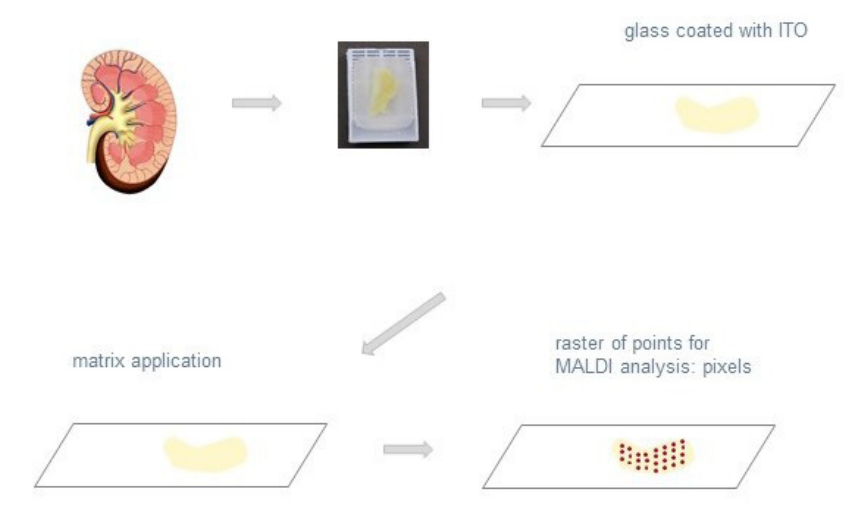

peptide peak pattern per pixel
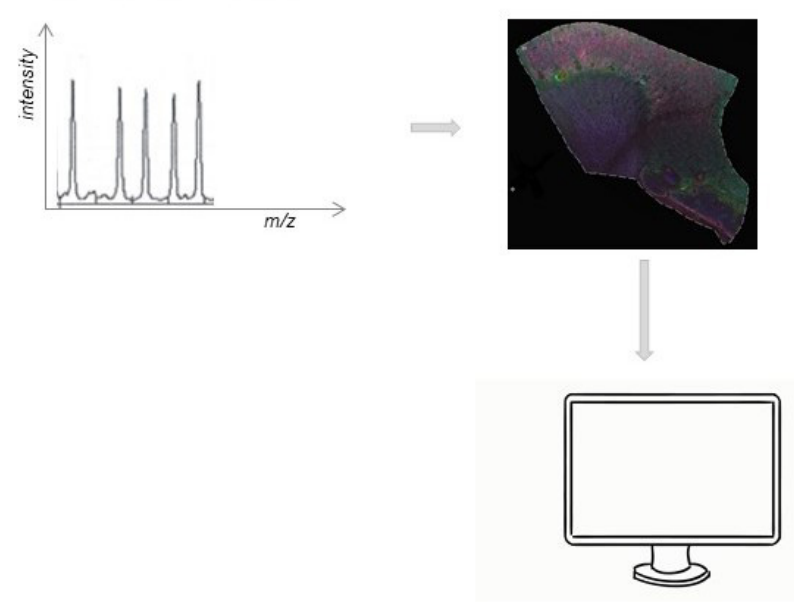

Figure 2 Workflow of MALDI IMS: the tissue is FFPE or frozen. Tissue sections around 5-10 $\mu \mathrm{m}$ thick are cut. Preparation steps differ, depending on the tissue used (FFPE: deparaffinization, washing with xylene to rewax, rehydration, antigen retrieval, trypsin solution and enzyme application; fresh frozen tissue: ethanol washing \pm trypsin application, depending on the molecular weight of the protein). The treated tissue is put on a conductive, transparent material, typically, a glass slide coated with ITO. A suitable matrix for desorption and ionization is applied onto the slide. For many points/pixels on the tissue, a mass spectrum is generated based on mass-to-charge ratio, in a time-of flight mass spectrometer device. An image can be constructed for each peak length (peptide) representing the relative abundance of the peptide on each pixel. FFPE, formalin-fixed, paraffin-embedded; ITO, indium-tin oxide; MALDI IMS, matrix-assisted laser desorption/ ionisation imaging mass spectrometry.

accelerated in a fixed electric field. Mass to charge ratio $(\mathrm{m} / \mathrm{z})$ is derived by determination of the time the ions travel through the fixed length of a flight tube. Ions with a lower $\mathrm{m} / \mathrm{z}$ ratio will have a higher speed and reach the detector faster than molecules with a larger $\mathrm{m} / \mathrm{z}$. After calibration, a plot of $\mathrm{m} / \mathrm{z}$ ratio ( $\mathrm{x}$-axis) and intensity (ion counts, y-axis) is achieved. This plot is the MS spectrum. The mass range in a normal MALDI-TOF instrument is in theory not limited, though usually in MALDI IMS it amounts to $30 \mathrm{kDa}$ for intact protein analysis in FF sections. For peptides obtained from FFPE section after digestion, the mass range is $500-3200 \mathrm{~m} / \mathrm{z}^{20}$ The mass spectra are recorded with their two-dimensional coordinates on the tissue slide and then are processed, and images with localization of the analysed molecules are reconstructed. MALDI is a so-called 'soft' ionisation process because the matrix is the actual energy absorber instead of the tissue itself, minimising the protein fragmentation and/or destruction. For a more detailed discussion of technical aspects, we refer to the literature. ${ }^{24-28}$

For alternative imaging MS techniques such as desorption electrospray ionisation (DESI), ${ }^{29}$ MALDI Fourier-transform ion cyclotron resonance MS imaging, ${ }^{30}$ laser ablation electrospray ionisation ${ }^{31} 32$ and electrospray-assisted desorption/ionisation, ${ }^{33}$ we equally refer to the literature.

\section{Some applications and future perspectives of MALDI IMS}

In the oncology field, publications have highlighted applications of MALDI IMS in lung cancer, breast cancer, brain tumours, gastrointestinal tract cancer, urogenital cancer, and head and neck cancer, among others. ${ }^{34-37}$ This technique has been experimentally applied for the detection of malignant tumour margins, tumour typing, grading ${ }^{37}$ and determination of cancer heterogeneity. ${ }^{21}$

Most papers are in the research phase, though some include potential applications in diagnostic decision-making, for example, for determination of primary carcinoma type in peritoneal or pleural fluid washes. ${ }^{38}$

Digital PCR (dPCR) with subsequent MALDI IMS has also been investigated. Performing proteomic and genetic analyses on the same tissue section is an attractive alternative when low amounts of tissue are available. Kazdal et al demonstrated MALDI-IMS did not deteriorate the genetic information for dPCR analysis. ${ }^{20}$

Outside of the oncology field, applications are scarcer though still promising. An example from the pathology lab is the use of a highly sensitive and cost-effective MS-based test system for the detection of human papillomaviruses in FFPE tissue sections. This MALDI IMS method is suitable for detection of both high-isk and low-risk human papilloma virus subtypes. ${ }^{39}$

\section{Advantages and disadvantages of MALDI IMS compared with IHC}

Both MALDI IMS and IHC are techniques to visualise proteins or peptides in the tissue, with preservation of histology. Therefore, there are similarities but also important differences.

MALDI IMS allows for an untargeted investigation of molecules in a section, enabling an unbiased analysis, since no antibodies are needed for detection. In IHC, often diagnostic inconsistencies result from variable purity and different specificity of the antibody. ${ }^{40}$ An immunohistochemical signal is at best semiquantitative. IHC signal intensity follows a steep logistical (sigmoidal) chart in the function of epitope concentration. This means that a certain epitope concentration is needed for a staining intensity to be present altogether, followed by a small window where staining intensity is almost linearly correlated to epitope concentration. Nonetheless, this linearity is quickly maxed out at higher antigen concentrations, inhibiting further possible quantification. ${ }^{41}$ In MALDI IMS, absolute quantification is equally difficult, but because of other reasons. Strong signal variations resulting from non-uniform crystal formation, co-crystallisation issues of matrix and analyte across the substrate and the difficulty of introducing standards in the tissue. Therefore, MALDI IMS is often considered as, at best, semiquantitative in the sense that relative differences in intensities of different analytes in the same pixel or region are taken into account. Nevertheless, several technical solutions do exist to collect absolute quantitative data from individual compounds 
in MALDI IMS, although these are, at the moment difficult to implement on a routine basis. ${ }^{42}$

In MALDI IMS, many different peptides can be analysed and located in a single assay at no additional cost in analysis time, labels or biological material. ${ }^{43}$ IHC is in comparison a rather low throughput technique, because, typically, one antigen is assayed on one slide at a time, though double or even triple staining for two and three antigens, respectively, on one slide has become more common. ${ }^{44}$ Still, performing a panel of IHC stains is at the cost of needing multiple slides using a considerable amount of tissue.

Many of the limitations of IHC extend to IF techniques, but some differences exist. IF is typically performed on frozen sections. As no formaldehyde is used for IHC or IF on frozen sections, there is no crosslinking of proteins and protein configuration is preserved. No antigen retrieval techniques are therefore applied and conduction time is fast. ${ }^{46}$ IF boasts an often higher sensitivity and specificity. ${ }^{47}$ However, morphology is hindered by freezing artefacts, and slides need to be stored in refrigerated conditions instead of room temperature. Additionally, an immunofluorescent microscope is needed for reading and interpretation of the slides, and this is sometimes seen as a drawback for routine practice in comparison with enzymatic visualisation techniques that make the immune reaction visible under a simple light microscope. However, multiplexing is easier to achieve in IF by using multiple antibodies, each tagged by a different fluorophore (different wavelength) or even by multispectral imaging. ${ }^{15}$

Both FF and FFPE samples can be assayed with MALDI, ${ }^{19}$ although analysis of FFPE needs more preparation steps and is generally more complex and less reproducible. ${ }^{48}$ The advantage of FFPE tissues is that they are routinely available from pathology archives spanning many years, which make them an ideal resource for retrospective biomarker discovery. ${ }^{19}$

In addition to the identity (through their molecular mass) of molecules, information on spatial distribution is obtained directly by scanning the laser in raster on the surface of the tissue. By recording both the spectra and the location from where the spectra were obtained, images can be created showing the distribution of individual molecules in the tissue. Relative abundances of a large number of biomolecules (eg, peptides, proteins and metabolites) can be provided directly in situ from a tissue specimen.

MALDI IMS is mostly non-destructive, this gives the possibility to use the tissue slide afterwards for conventional histochemical or immunohistochemical imaging or other molecular analyses (such as PCR). ${ }^{20}$ Even though MALDI IMS provides a high level of molecular information, a good spatial resolution is generally at the cost of high data acquisition times and sensitivity, although recently there has been progress on the matter. ${ }^{49}$ The combination of the two techniques, microscopy and MALDI-IMS, results in an image with good spatial resolution and high molecular specificity. ${ }^{50}$

Both MALDI IMS and digitally scanned IHC slides are amenable to machine learning. ${ }^{15} 51-53$

MS instruments are not yet in the standard equipment of the average pathology laboratory. The instrument time, though declining, can be long and instruments are expensive. However, recent studies performed with high-speed mass spectrometers demonstrate a total measurement time of 1.5 hours per tissue at a $50 \mu \mathrm{m}$ pixel size for tissue sections of $\pm 5 \mu \mathrm{m}$ thickness. ${ }^{21}$ Recent years have seen a breakthrough in the use of cheaper 'desktop' mass spectrometers for microbiology, indicating that these systems are increasingly around in hospitals and medical laboratories. $^{54}$
Table 1 Comparison of properties of immunohistochemistry and MALDI imaging for protein analysis

\begin{tabular}{|c|c|c|}
\hline & Immunohistochemistry & MALDI imaging \\
\hline Spatial information & $\begin{array}{l}\text { Localisation of protein/peptide } \\
\text { epitopes }\end{array}$ & $\begin{array}{l}\text { Localisation of proteins/ } \\
\text { peptides }\end{array}$ \\
\hline Antibodies & $\begin{array}{l}\text { Costly generation of antibodies, } \\
\text { not available for all antigens }\end{array}$ & $\begin{array}{l}\text { No antibodies needed: } \\
\text { label free }\end{array}$ \\
\hline Tissue thickness & $2-5 \mu \mathrm{m}$ slides & $\begin{array}{l}\text { Typically, 5-10 } \mu \mathrm{m} \text { slides } \\
\text { needed }\end{array}$ \\
\hline Throughput & $\begin{array}{l}\text { Low: one to three antibodies } \\
\text { at a time }\end{array}$ & $\begin{array}{l}\text { High: up to more than } \\
100 \text { peptides at a time }\end{array}$ \\
\hline Tissue type & FFPE and frozen tissue & FFPE and frozen tissue \\
\hline Destructiveness & Non-destructive & Non-destructive \\
\hline Preanalytic factors & Need to be controlled & $\begin{array}{l}\text { Need to be controlled } \\
\text { Tissue dependent }\end{array}$ \\
\hline Laboratory procedures & Interlaboratory variability & $\begin{array}{l}\text { No standardised } \\
\text { laboratory procedures } \\
\text { yet }\end{array}$ \\
\hline Interpretation & $\begin{array}{l}\text { Human eye (interobserver } \\
\text { variability), unless computer } \\
\text { qualification on digital slides }\end{array}$ & $\begin{array}{l}\text { Computer evaluation (no } \\
\text { interobserver variability) }\end{array}$ \\
\hline Quantification & Semiquantitative & Semiquantitative \\
\hline
\end{tabular}

FFPE, formalin-fixed, paraffin-embedded; MALDI, matrix-assisted laser desorption/ ionisation.

Considering technical notes, software and an overview of commercial instruments, information can be found at the website of the MS Imaging Society (https://ms-imaging.org).

For a summary of characteristics of MALDI IMS compared with IHC, we refer to table 1.

\section{Example of a candidate disease to evaluate by MALDI IMS: amyloidosis}

Amyloidosis is a group of disorders characterised by abnormal protein folding. The conformational change that occurs in susceptible proteins leads to the formation of an insoluble betapleated sheet secondary structure. ${ }^{55}$ This misfolding causes extracellular accumulation of pathological amyloid protein. Because these proteins are abundant and concentrated in the depositions, amyloidosis is a good candidate for analysis by MALDI IMS as signal to noise ratio is expected to be high.

Furthermore, from a clinical perspective, amyloidosis represents a disease in which an improvement in diagnostic accuracy has huge clinical consequences, that is, better treatment options and thus prognosis. Improving the knowledge on newer diagnostic techniques in a broad audience of pathologists might also set the scene for clinical implementation of MALDI IMS.

The most common forms of amyloidosis are immunoglobulin light-chain (AL) amyloidosis and amyloid A (AA) amyloidosis. The latter is a secondary form in various chronic inflammatory diseases. Hereditary amyloidosis is a third group. ${ }^{56}$

Currently, conventional microscopy and IHC are typically used to diagnose amyloidosis. Amyloid deposits stained by Congo Red show apple-green birefringence under polarised light. IHC studies are helpful in subtyping, using a more or less extensive panel of stains. ${ }^{55}$ However, laboratories typically do not have antibodies available for detection of all amyloid subtypes. ${ }^{55} \mathrm{MS}$ in general is a very valuable and straightforward alternative as it does not require target-specific antibodies and has the ability to identify multiple different peptides at once, including new and unknown ones. ${ }^{57}$

Interestingly, MS can even be used upfront as it detects subtle amyloid deposition in an earlier stage than Congo red staining. ${ }^{57}$ The MS diagnosis of amyloidosis is based on detection of 
serum amyloid P component, apolipoprotein E, vitronectin and perlecan since all of these proteins are linked to both AL and AA amyloidoses. ${ }^{56-59}$ Although mostly tissue extraction and liquid chromatography (LC)-based MS techniques have been used for analysis of amyloid, ${ }^{56}$ MALDI IMS might be very suitable as well. An important advantage of MALDI IMS is the preservation of spatial information since one does not need to make a tissue lysate as in LC-based MS. ${ }^{60}$

\section{OTHER TECHNIQUES}

A technique called mass spectrometry immunohistochemistry (MSIHC) combines IHC with MS. ${ }^{61-63}$

Instead of fluorophores or chromogens, metal-labelled antibodies are used for detection of peptides of interest. The strategy employs antibodies that are labelled with elemental mass tags, such as isotopically pure lanthanides not typically found in biological specimens, rather than with typical fluorophores or chromogens. The metal-labelled antibodies are then detected in tissue using lasers or ion beams to liberate the tags for subsequent MS detection. Typically, magnetic sector instruments are time-of flight (TOF) instruments that are dedicated just for this type of analysis. In a multiplex panel, the metal labels are selected so as not to overlap. In theory, this means up to 100 antibodies (for respective antigens) can be detected at once on a single slide. ${ }^{61}$

In the TagMass method, a specifically designed antibody against a target antigen ${ }^{6465}$ is linked to a laser-cleavable peptide ${ }^{64-68}$ with known sequence. ${ }^{6465}$ An MS image of the tissue is generated. The sequence and the identity of the peptide are then determined by means of MS and are subsequently imaged on the surface of the tissue $^{65-67}$ In contrast to MSIHC, this type of analysis can be performed with fairly common MALDI IMS instruments.

Scanning mass cytometry (SMC) is another technique in which a form of laser ablation technique with spot sizes down to $1 \mathrm{~mm}$ is used to liberate tissue into a carrier gas. The ablated sample is then transported into argon plasma, ionised and thereafter detected by MS. All targets are scanned at once, while the full sample thickness is destroyed. ${ }^{6162}$

Multiplexed ion-beam imaging (MIBI) is based on another MS technique than MALDI, namely, secondary ion mass spectrometry, in which the sample is scanned by an ion beam with a submicron spot size. A mass spectrometer then detects the released secondary ions. $^{6163}$

An alternative method is based on the combination of MALDI IMS with IHC, but without the need for the costly design and production of specific antibodies. Therefore, in situ tryptic digestion of the primary antibody against an antigen is imaged. This tryptic digestion is needed, due to the large masses of the primary native antibodies. Furthermore, it is also possible to separate the heavy chains from the light chains by means of reduction and alkylation. Instead of the application of a secondary antibody, a matrix layer is applied after digestion an overnight incubation for the desorption-ionisation of the digested fragments of the primary antibody. The principle consists of a direct comparison of the peptide mass fingerprint after in-gel tryptic digestion and the peptide mass fingerprint after antibody incubation, followed by tryptic digestion. A great advantage of this method is the compatibility with every polyclonal and monoclonal antibody for the detection of a specific antigen, whereas Tag Mass, SMC and MIBI need specific designs of antibody and labelling. ${ }^{65}$

All these techniques allow for extensive multiplexing and are considered a 'next-generation' approach to IHC. 6169 The most limiting factor of MSIHC is the cost, ${ }^{61}$ including design of antibodies and cost of instruments for analysis and imaging.
Vibrational spectroscopy is another group of innovative techniques that can detect molecular changes in tissue. Raman spectroscopy and Fourier transform infrared microspectroscopy are both vibrational imaging modalities. Here, imaging is based on physical characteristics of the interaction between light and matter providing data based on the molecular vibrations on the chemical bonds between atoms. Although the principle is not new, due to great advances in instruments, high-quality images can be produced. Just as for MALDI IMS, spectral histopathology is based on the detection of changes in biochemical composition, rather than on morphological features, and shows that tissue morphological features observed in classical pathology are accompanied by changes in the biochemical composition at the cellular level. ${ }^{70} 71$

\section{CONCLUSION}

Although MALDI IMS is often complex and requires investments in equipment, applications might be coming into the routine pathology laboratory relatively soon, probably linked with software for unbiased image analysis and deep learning.

There is evidently a great need for multiplex systems that have the ability to measure several proteins at once. Oncology might benefit from techniques where predictive biomarkers are quickly analysed in parallel. In the non-oncological field, the imaging and characterisation of amyloid in tissues by MALDI IMS are one promising example.

DNA markers have made their way into our laboratories over the past few decades, first detected by PCR, then by Sanger sequencing and massive parallel sequencing (next-generation sequencing); it might just be about time for proteins to follow course.

\section{Handling editor Tahir S Pillay.}

Contributors DA designed the initial structure of the review. AM, BG, DA, VCAH and $B G$ edited and reviewed the manuscript. GB and PP reviewed the manuscript. SK performed the MALDI experiment to generate a picture of a human kidney.

Funding The authors have not declared a specific grant for this research from any funding agency in the public, commercial or not-for-profit sectors.

Competing interests None declared.

Patient consent for publication Not required.

Provenance and peer review Not commissioned; externally peer reviewed.

\section{ORCID iDs}

Melek Ahmed http://orcid.org/0000-0001-5573-9353

Glenn Broeckx http://orcid.org/0000-0002-7543-8061

\section{REFERENCES}

1 van den Tweel JG, Taylor CR. Introduction to the History of Pathology series. Virchows Arch 2010;457:1-2

2 Moore DA, Young CA, Morris HT, et al. Time for change: a new training programme for morpho-molecular pathologists? J Clin Pathol 2018;71:285-90.

3 Graves PR, Haystead TAJ. Molecular biologist's guide to proteomics. Microbiol Mol Biol Rev 2002;66:39-63.

4 Dabbs D. Diagnostic Immunohistochemistry: Theranostic and Genomic Applications. 5th edn. Elsevier, 2018.

5 Raju TN. The Nobel chronicles. 1984: Niels Kai Jerne, (1911-94); César Milstein (b 1926); and Georges Jean Franz Köhler (1946-95). Lancet 2000;355:75.

6 Prichard JW. Overview of automated immunohistochemistry. Arch Pathol Lab Med 2014;138:1578-82

7 Gosselin EJ, Cate CC, Pettengill OS, et al. Immunocytochemistry: its evolution and criteria for its application in the study of epon-embedded cells and tissue. Am J Anat 1986;175:135-60.

8 Shi S-R, Liu C, Pootrakul L, et al. Evaluation of the value of frozen tissue section used as "gold standard" for immunohistochemistry. Am J Clin Pathol 2008;129:358-66.

9 Coons $\mathrm{AH}$, Creech HJ, Jones RN. Immunological properties of an antibody containing a fluorescent group. Exp Biol Med 1941;47:200-2.

10 Senkus E, Kyriakides S, Ohno S, et al. Primary breast cancer: ESMO Clinical Practice Guidelines for diagnosis, treatment and follow-up. Ann Oncol 2015;26 Suppl 5:v8-30.

11 Kerr K, Bubendorf L, Edelman MJ, et al. ESMO Consensus Guidelines: Pathology and molecular biomarkers for non-small-cell lung cancer. Ann Oncol 2014;25:1681-90. 
12 Dummer R, Hauschild A, Guggenheim M, et al. Cutaneous melanoma: ESMO Clinical Practice Guidelines for diagnosis, treatment and follow-up. Ann Oncol 2012;23 Suppl 7:viï6-91.

13 Mohsin SK, Weiss H, Havighurst T, et al. Progesterone receptor by immunohistochemistry and clinical outcome in breast cancer: a validation study. Mod Pathol 2004;17:1545-54.

14 Harvey JM, Clark GM, Osborne CK, et al. Estrogen receptor status by immunohistochemistry is superior to the ligand-binding assay for predicting response to adjuvant endocrine therapy in breast cancer. JCO 1999;17:1474-81.

15 Madabhushi A, Lee $\mathrm{G}$. Image analysis and machine learning in digital pathology: Challenges and opportunities. Med Image Anal 2016;33:170-5.

16 Lippincott-Schwartz J. Profile of Eric Betzig, Stefan Hell, and W. E. Moerner, 2014 Nobel Laureates in Chemistry. Proc Natl Acad Sci U S A 2015;112:2630-2.

17 Weber M, Mickoleit M, Huisken J. Light sheet microscopy. Methods Cell Biol 2014:123:193-215.

18 Caprioli RM, Farmer TB, Gile J. Molecular imaging of biological samples: localization of peptides and proteins using MALDI-TOF MS. Anal Chem 1997;69:4751-60.

19 Hoffmann F, Umbreit C, Krüger T, et al. Identification of Proteomic Markers in Head and Neck Cancer Using MALDI-MS Imaging, LC-MS/MS, and Immunohistochemistry. Proteomics Clin Appl 2019;13:1700173.

20 Kazdal D, Longuespée R, Dietz S, et al. Digital PCR After MALDI-Mass Spectrometry Imaging to Combine Proteomic Mapping and Identification of Activating Mutations in Pulmonary Adenocarcinoma. Proteomics Clin Appl 2019:13:1800034.

21 Kriegsmann J, Kriegsmann M, Kriegsmann K, et al. MALDI Imaging for Proteomic Painting of Heterogeneous Tissue Structures. Proteomics Clin Appl 2019;13:1800045.

22 Casadonte R, Caprioli RM. Proteomic analysis of formalin-fixed paraffin-embedded tissue by MALDI imaging mass spectrometry. Nat Protoc 2011;6:1695-709.

23 Zubair F, Laibinis PE, Swisher WG, et al. Trypsin and MALDI matrix pre-coated targets simplify sample preparation for mapping proteomic distributions within biological tissues by imaging mass spectrometry. J Mass Spectrom 2016;51:1168-79.

24 Hortin GL. The MALDI-TOF mass spectrometric view of the plasma proteome and peptidome. Clin Chem 2006;52:1223-37

25 Gustafsson JOR, Oehler MK, Ruszkiewicz A, et al. MALDI Imaging Mass Spectrometry (MALDI-IMS)-application of spatial proteomics for ovarian cancer classification and diagnosis. Int J Mol Sci 2011;12:773-94.

26 Franck J, Arafah K, Elayed M, et al. MALDI Imaging Mass Spectrometry. Mol Cell Proteomics 2009:8:2023-33.

27 Franck J, Longuespée R, Wisztorski M, et al. MALDI mass spectrometry imaging of proteins exceeding 30,000 daltons. Med Sci Monit 2010;16:BR293-BR299.

28 Gessel MM, Norris JL, Caprioli RM. MALDI imaging mass spectrometry: spatial molecular analysis to enable a new age of discovery. J Proteomics 2014;107:71-82.

29 Alfaro CM, Jarmusch AK, Pirro V, et al. Ambient ionization mass spectrometric analysis of human surgical specimens to distinguish renal cell carcinoma from healthy renal tissue. Anal Bioanal Chem 2016:408:5407-14.

30 Piga I, Heijs B, Nicolardi S, et al. Ultra-high resolution MALDI-FTICR-MSI analysis of intact proteins in mouse and human pancreas tissue. Int J Mass Spectrom 2019;437:10-16.

31 Kiss A, Smith DF, Reschke BR, et al. Top-down mass spectrometry imaging of intact proteins by laser ablation ESI FT-ICR MS. Proteomics 2014;14:1283-9.

32 Nemes P, Vertes A. Laser ablation electrospray ionization for atmospheric pressure, in vivo, and imaging mass spectrometry. Anal Chem 2007;79:8098-106.

33 Huang M-Z, Hsu H-J, Lee J-Y, et al. Direct protein detection from biological media through electrospray-assisted laser desorption ionization/mass spectrometry. J Proteome Res 2006;5:1107-16.

34 Torata N, Kubo M, Miura D, et al. Visualizing Energy Charge in Breast Carcinoma Tissues by MALDI Mass-spectrometry Imaging Profiles of Low-molecular-weight Metabolites. Anticancer Res 2018:38:4267-72.

35 Erich K, Reinle K, Müller T, et al. Spatial distribution of endogenous tissue protease activity in gastric carcinoma mapped by MALDI mass spectrometry imaging. $\mathrm{Mo} / \mathrm{Cell}$ Proteomics 2019;18

36 Marko-Varga G, Fehniger TE, Rezeli M, et al. Drug localization in different lung cancer phenotypes by MALDI mass spectrometry imaging. J Proteomics 2011;74:982-92.

37 Kriegsmann J, Kriegsmann M, Casadonte R. MALDI TOF imaging mass spectrometry in clinical pathology: a valuable tool for cancer diagnostics (review). Int J Oncol 2015;46:893-906

38 Schwamborn K, Weirich G, Steiger K, et al. Discerning the Primary Carcinoma in Malignant Peritoneal and Pleural Effusions Using Imaging Mass Spectrometry-A Feasibility Study. Proteomics Clin App/ 2019;13:e1800064.

39 Kriegsmann M, Wandernoth P, Lisenko K, et al. Detection of HPV subtypes by mass spectrometry in FFPE tissue specimens: a reliable tool for routine diagnostics. J Clin Pathol 2017;70:417-23.

40 Zeng Z, Zhang P, Zhao N, et al. Using oligonucleotide aptamer probes for immunostaining of formalin-fixed and paraffin-embedded tissues. Mod Pathol 2010;23:1553-8.

41 Thunnissen E, de Langen AJ, Smit EF. PD-L1 IHC in NSCLC with a global and methodological perspective. Lung Cancer 2017;113:102-5.
42 Rzagalinski I, Volmer DA. Quantification of low molecular weight compounds by MALDI imaging mass spectrometry - A tutorial review. Biochim Biophys Acta Proteins Proteom 2017; 1865:726-39.

43 Chaurand P, Norris JL, Cornett DS, et al. New developments in profiling and imaging of proteins from tissue sections by MALDI mass spectrometry. J Proteome Res 2006;5:2889-900.

44 Daoud NA, Li G, Evans AJ, et al. The value of triple antibody $(34 \beta E 12+$ p63 + AMACR) cocktail stain in radical prostatectomy specimens with crushed surgical margins. J Clin Pathol 2012;65:437-40.

45 Stack EC, Wang C, Roman KA, et al. Multiplexed immunohistochemistry, imaging, and quantitation: a review, with an assessment of Tyramide signal amplification, multispectral imaging and multiplex analysis. Methods 2014;70:46-58.

46 Chaudhary M, Jain D, Gawande M, et al. A Comparative Study between IHC in Frozen Sections and Formalin Fixed Sections and their Clinical Significance - A Retrospective Study. J Denst Otolaryngol 2014;14

47 Allaire A, Picard-Jean F, Bisaillon M. Immunofluorescence to Monitor the Cellular Uptake of Human Lactoferrin and its Associated Antiviral Activity Against the Hepatitis C Virus. J Vis Exp 2015;(104). doi:10.3791/53053.

48 Lalowski M, Magni F, Mainini V, et al. Imaging mass spectrometry: a new tool for kidney disease investigations. Nephrol Dial Transplant 2013;28:1648-56.

49 Smith A, L'Imperio V, Denti V, et al. High Spatial Resolution MALDI-MS Imaging in the Study of Membranous Nephropathy. Proteomics Clin App/ 2019;13:1800016.

50 Caprioli RM. Imaging mass spectrometry: Molecular microscopy for the new age of biology and medicine. Proteomics 2016:16:1607-12.

51 Galli M, Zoppis I, Smith A, et al. Machine learning approaches in MALDI-MSI: clinical applications. Expert Rev Proteomics 2016;13:685-96.

52 Ganggayah MD, Taib NA, Har YC, et al. Predicting factors for survival of breast cancer patients using machine learning techniques. BMC Med Inform Decis Mak 2019;19:48.

53 Attuluri AK, Serkad CPV, Gunda A, et al. Analytical validation of CanAssist-Breast: an immunohistochemistry based prognostic test for hormone receptor positive breast cancer patients. BMC Cancer 2019;19:249.

54 Tan KE, Ellis BC, Lee $\mathrm{R}$, et al. Prospective evaluation of a matrix-assisted laser desorption ionization-time of flight mass spectrometry system in a hospital clinical microbiology laboratory for identification of bacteria and yeasts: a bench-by-bench study for assessing the impact on time to identification and cost-effectiveness. J Clin Microbiol 2012;50:3301-8.

55 Picken MM. Proteomics and mass spectrometry in the diagnosis of renal amyloidosis. Clin Kidney J 2015:8:665-72.

56 Sethi S, Vrana JA, Theis JD, et al. Laser microdissection and mass spectrometrybased proteomics aids the diagnosis and typing of renal amyloidosis. Kidney In 2012;82:226-34

57 Casadonte R, Kriegsmann M, Deininger S-O, et al. Imaging mass spectrometry analysis of renal amyloidosis biopsies reveals protein co-localization with amyloid deposits. Anal Bioanal Chem 2015:407:5323-31.

58 Hill MM, Mollee PN. Mass spectrometry analysis for amyloidosis typing - is the future bright for its clinical implementation? Expert Rev Proteomics 2017:14:565-6.

59 Lavatelli F, Merlini G. Proteomics with Mass Spectrometry Imaging: Beyond Amyloid Typing. Proteomics 2018;18:1700353.

60 Winter M, Tholey A, Kristen A, et al. MALDI Mass Spectrometry Imaging: A Novel Tool for the Identification and Classification of Amyloidosis. Proteomics 2017:17:1700236.

61 Levenson RM, Borowsky AD, Angelo M. Immunohistochemistry and mass spectrometry for highly multiplexed cellular molecular imaging. Lab Invest 2015;95:397-405.

62 Giesen C, Wang HAO, Schapiro D, et al. Highly multiplexed imaging of tumor tissues with subcellular resolution by mass cytometry. Nat Methods 2014;11:417-22.

63 Angelo M, Bendall SC, Finck R, et al. Multiplexed ion beam imaging of human breast tumors. Nat Med 2014;20:436-42.

64 Gagnon H, Franck J, Wisztorski M, et al. Targeted mass spectrometry imaging: specific targeting mass spectrometry imaging technologies from history to perspective. Prog Histochem Cytochem 2012:47:133-74.

65 Longuespée R, Boyon C, Desmons A, et al. Spectroimmunohistochemistry: a novel form of MALDI mass spectrometry imaging coupled to immunohistochemistry for tracking antibodies. OMICS 2014;18:132-41.

66 Lemaire R, Stauber J, Wisztorski M, et al. Tag-mass: specific molecular imaging of transcriptome and proteome by mass spectrometry based on photocleavable tag. J Proteome Res 2007;6:2057-67.

67 Lemaire R, Lucot J, Collinet $\mathrm{P}$, et al. New developments in direct analysis by MALDI mass spectrometry for the study of ovarian cancer. Mol Cell Proteomics 2005:4:S306-S.

68 Stauber J, El Ayed M, Wisztorski M, et al. Polymerase chain reaction and immunoassay--matrix assisted laser desorption mass spectrometry using tag-mass technology: new tools to break down quantification limits and multiplexes. Anal Chem 2009:81:9512-21.

69 Rimm DL. Next-gen immunohistochemistry. Nat Methods 2014;11:381-3

70 Akalin A, Ergin A, Remiszewski S, et al. Resolving Interobserver Discrepancies in Lung Cancer Diagnoses by Spectral Histopathology. Arch Pathol Lab Med 2019;143.

71 Mittal S, Yeh K, Leslie LS, et al. Simultaneous cancer and tumor microenvironment subtyping using confocal infrared microscopy for all-digital molecular histopathology. Proc Natl Acad Sci U SA 2018;115:E5651-E5660. 